\title{
Delayed administration of guanosine improves long-term functional recovery and enhances neurogenesis and angiogenesis in a mouse model of photothrombotic stroke
}

\author{
GANG DENG, ZHANDONG QIU, DAYONG LI, YU FANG and SUMING ZHANG \\ Department of Neurology, Tongji Hospital, Tongji Medical College, Huazhong University \\ of Science and Technology, Wuhan, Hubei 430030, P.R. China
}

Received January 12, 2016; Accepted February 14, 2017

DOI: $10.3892 / \mathrm{mmr} .2017 .6521$

\begin{abstract}
Guanosine (GUO) is neuroprotective when administered acutely for the treatment of cerebral ischemia. The aim of the present study was to investigate whether delayed administration of GUO improved long-term functional recovery following stroke, as well as to explore the potential underlying mechanisms. GUO $(8 \mathrm{mg} / \mathrm{kg})$ or a vehicle was administered intraperitoneally for 7 consecutive days beginning $24 \mathrm{~h}$ prior to photothrombosis-induced stroke in male C57/B6J mice. Behaviour tests were performed at days 1, 3, 7, 14 and 28 post-stroke. Infarct volume was measured using Nissl staining at day 7 post-stroke. Neurogenesis and angiogenesis were evaluated by co-labelling bromodeoxyuridine (BrdU) with doublecortin (DCX), neuronal nuclei (NeuN) and von Willebrand factor, in immunohistochemical studies. Brain-derived neurotrophic factor (BDNF) and vascular endothelial growth factor (VEGF) levels in the ipsilesional brain at day 28 post-stroke were detected by western blot analysis. Delayed administration of GUO did not reduce infarct volume or affect neurological function at day 7 post-stroke; however, it did improve functional recovery from day 14 post-stroke, when compared with the vehicle group. GUO significantly increased the number of $\mathrm{BrdU}^{+}$and $\mathrm{BrdU}^{+} / \mathrm{DCX}^{+}$cells in the subventricular zone and subgranular zone at all examined time points, the number of $\mathrm{Brdu}^{+} / \mathrm{NeuN}^{+}$cells in the peri-infarction region at days 14 and 28 post-stroke and microvessel density in the peri-infarction region at day 28 post-stroke compared with the vehicle group. In addition, the BDNF and VEGF levels in the ipsilesional brain were significantly elevated. Delayed administration of GUO at
\end{abstract}

Correspondence to: Professor Suming Zhang, Department of Neurology, Tongji Hospital, Tongji Medical College, Huazhong University of Science and Technology, 1095 Jiefang Avenue, Wuhan, Hubei 430030, P.R. China

E-mail: sumingzhang123@163.com

Key words: guanosine, stroke, neurogenesis, angiogenesis
$24 \mathrm{~h}$ post-stroke enhanced neurogenesis and angiogenesis, and increased BDNF and VEGF levels, which likely contributes to long-term functional recovery following stroke.

\section{Introduction}

Stroke is a major cause of death and disability worldwide, however treatment options remain limited $(1,2)$. A small fraction of patients benefit from administration of recombinant tissue-type plasminogen activator; however, this has a short time window, as well as additional limitations (3) In a previous study, brain remodelling and plasticity, including neurogenesis and angiogenesis, has emerged as a novel promising therapeutic target for stroke (4). Persistent neurogenesis in the subventricular zone (SVZ) of the lateral ventricle and the subgranular zone (SGZ) of the dentate gyrus may be stimulated by cerebral ischemia and other injuries as part of the endogenous repair response (5). Angiogenesis, the formation of new microvessels from the existing vasculature, improves tissue microperfusion in the peri-infarction region following a stroke (6).

Guanosine (GUO), a guanine-based purine, serves several important roles in the central nervous system $(7,8)$. Endogenous GUO levels increase within $2 \mathrm{~h}$ of focal stroke and remain elevated for 7 days (9). An accumulating body of evidence indicates that exogenous administration of GUO prior to or immediately following experimental stroke confers acute neuroprotection following cerebral ischemia (10-14). However, any benefits to an ischemic stroke from delayed treatment of GUO remain unknown. Notably, GUO has been reported to promote neurite outgrowth from PC12 cell cultures (15) and proliferation of neural stem cells (16). In addition, GUO has been indicated to promote myelination in a murine model of spinal cord injury (17) and induce synaptogenesis in the brain of healthy animals (18). These results suggest that GUO may serve as a restorative target.

Therefore, the present study involved investigation of whether delayed treatment with GUO ( $24 \mathrm{~h}$ following stroke) improves the long-term functional outcome following a stroke, as well as exploring the possible mechanisms underlying GUO restorative effects. 


\section{Materials and methods}

Animals and experimental model of photothrombotic stroke. The experiments were approved by the Institutional Animal Care and Use Committee of Tongji Medical College, Huazhong University of Science and Technology (Wuhan, China), and were in accordance with the guidelines of the Institute of Laboratory Animal Resources (Washington, DC, USA). A total of 120 male C57BL/6J wild-type mice (weight, 20-25 g; age, 8-10 weeks old) were purchased from the Tongji Medical College Experimental Animal Centre (Wuhan, China). Animals were housed in a temperature- and humidity-controlled environment with a $12 \mathrm{~h}$ light/dark cycle and free access to food and $\mathrm{H}_{2} \mathrm{O}$. Focal stroke was induced by photothrombosis (PT) as described previously (19). Mice were anesthetized using $10 \%$ chloral hydrate (Sigma-Aldrich;Merck KGaA,Darmstadt,Germany) (35 mg/kg) intraperitoneally and placed in a stereotactic apparatus. A midline incision of the skin exposed the skull. A dose of $0.1 \mathrm{ml}$ rose bengal solution $(10 \mathrm{mg} / \mathrm{ml}$ in normal saline; Sigma-Aldrich; Merck KGaA) was injected intraperitoneally 5 min prior to illumination. For illumination, a cold light source (KL1500 LCD; Zeiss AG, Oberkochen, Germany) with a 4-mm aperture was centred $2 \mathrm{~mm}$ lateral from the bregma. The brain was illuminated through the intact skull for $15 \mathrm{~min}$. All mice survived the procedure and exhibited behavioural deficits.

Drug administration. All animals subjected to PT-induced stroke were randomly assigned to receive GUO or vehicle following stroke induction. Researchers were blinded to experimental groups. GUO $(0.5 \mathrm{mg} / \mathrm{ml}$ in sterile saline; Sigma-Aldrich; Merck KGaA) was administered intraperitoneally (i.p.; $8 \mathrm{mg} / \mathrm{kg}$ ) beginning $24 \mathrm{~h}$ following the stroke and then daily for 7 days. The vehicle group received an equal volume of saline. Bromo-deoxyuridine (BrdU; $10 \mathrm{mg} / \mathrm{ml}$ in sterile saline; Sigma-Aldrich; Merck KGaA) was injected i.p. (100 mg/kg) beginning $24 \mathrm{~h}$ following the stroke and then twice daily until the animals were sacrificed.

Measurement of infarct volume. The animals were sacrificed 7 days post-stroke. Anesthetized mice were transcardially perfused with cold PBS followed by $4 \%$ paraformaldehyde. Brains were subsequently removed, fixed in fresh $4 \%$ formaldehyde solution at $4{ }^{\circ} \mathrm{C}$ overnight and immersed in $30 \%$ sucrose until they sank. Then the brains were frozen at $-80^{\circ} \mathrm{C}$. The frozen brains were cut into $10-\mu \mathrm{m}$ coronal sections on a cryostat (CM3050S; Leica Microsystems GmbH, Wetzlar, Germany). For each brain, 15 sequential sections were taken at $100-\mu \mathrm{m}$ intervals and processed for Nissl staining. Sections were stained with $0.1 \%$ crystal violet solution for $10 \mathrm{~min}$ at room temperature. Infarct volumes were measured using an image analysis program (ImageJ version 1.46r; National Institutes of Health, Bethesda, MD, USA).

Behavioural testing. Behavioural tests were carried out prior to the PT procedure and 1, 7, 14 and 28 days following PT using the modified neurological severity scale (mNSS), grid walking test and cylinder test.

$m N S S$. mNSS is a composite of motor, sensory, balance and reflex tests. Neurological function is graded on a scale of 0 to
18 (normal score, 0 ; maximal deficit score, 18). A total of 1 score point is awarded for the inability to perform the test or for the lack of a tested reflex; therefore, a more severe injury has an increased score (20).

Grid walking test. Animals were placed on an elevated wire grid and video-recorded when they walked. The number of contra- and ipsilateral faults for each limb and the total number of steps taken were counted, the ratio between foot faults and total steps was calculated (21).

Cylinder test. The animal was placed in a transparent cylinder and video-recorded. Forelimb preference during vertical exploration of the cylinder was evaluated by recording the forelimb contacts. Animals were subjected to one trial on day 1 prior to PT. The asymmetry score for each animal was calculated by the formula previously described (22).

Immunohistochemistry. Frozen sections were incubated with a blocking buffer (1X PBS/5\% normal goat serum/0.3\% Triton $\mathrm{X}-100$, Goodbio, Wuhan, China) for $1 \mathrm{~h}$ at room temperature. The sections were subsequently incubated at $4^{\circ} \mathrm{C}$ overnight with the following primary antibodies: mouse monoclonal anti-BrdU (catalogue no. sc-32323, dilution, 1:100, Santa Cruz Biotechnology, Inc., Dallas, TX, USA), rabbit polyclonal anti-BrdU (catalogue no. ab19944, dilution, 1:500, Abcam, Cambridge, MA, USA), goat polyclonal anti-doublecortin (DCX; catalogue no. sc-8066, dilution, 1:200; Santa Cruz Biotechnology, Inc.), mouse monoclonal neuronal nuclei (NeuN; Catalogue no. MAB377, dilution, 1:500; EMD Millipore, Billerica, MA, USA) and von Willebrand factor (vWF; catalogue no. ab7356, dilution, 1:1,000; EMD Millipore). Following washing with PBS, the sections were incubated for $1 \mathrm{~h}$ at room temperature with two secondary antibodies: Alexa Fluor 488 (catalogue no. CA11001s, dilution, 1:200; Invitrogen; Thermo Fisher Scientific, Inc., Waltham, MA, USA) and Alexa Fluor 594 (catalogue no. CA11012s, dilution, 1:200; Invitrogen; Thermo Fisher Scientific, Inc.). Incubation was conducted in the dark and then washed with PBS three times. For BrdU immunofluorescence, brain sections were pre-treated with $2 \mathrm{M} \mathrm{HCl}$ at $37^{\circ} \mathrm{C}$ for $30 \mathrm{~min}$ and then washed with PBS six times at room temperature before being incubated with blocking solution. The images were captured using a fluorescence microscope (BX51; Olympus Corporation, Tokyo, Japan).

Western blot. Mice were anesthetized and decapitated at 14 days post-stroke. The ipsilateral peri-infarct cortices and cognate regions from the contralateral hemisphere were sampled. The total protein of each tissue was extracted according to the instructions of the protein reagent kit (Goodbio, Wuhan, China). Protein concentration of each sample was determined using the bicinchoninic acid assay (Sigma-Aldrich; EMD Millipore). Protein samples (20 $\mu$ g per lane) were subsequently separated by $10 \%$ SDS-PAGE and transferred to polyvinylidene difluoride membranes. Two primary antibodies were incubated with the membranes overnight at $4^{\circ} \mathrm{C}$ : anti-brain-derived neurotrophic factor (BDNF; catalogue no. AB1779, dilution, 1:200; EMD Millipore), anti-vascular endothelial growth factor (VEGF; catalogue no. sc-152, dilution, 1:200; Santa 

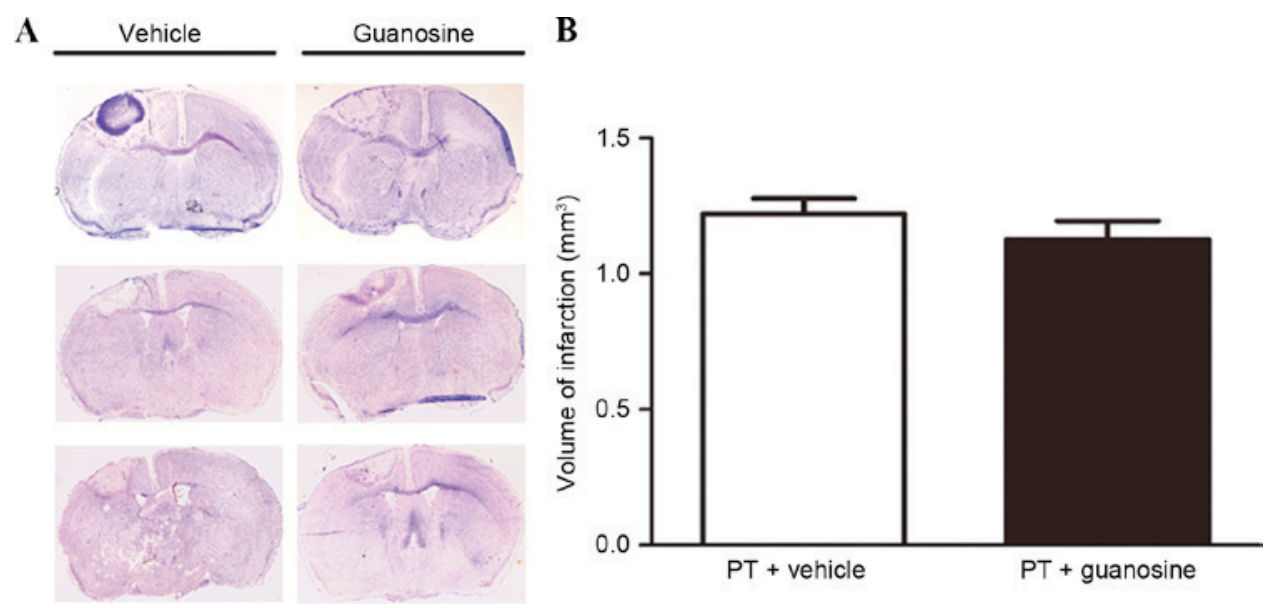

Figure 1. Delayed administration of guanosine does not reduce the infarct volume at day 7 following PT-induced stroke. (A) Representative Nissl stained sections from vehicle- and guanosine-treated mice following 7 days of stroke. (B) Quantification of the infarct volume of vehicle and guanosine group. Data are presented as the mean \pm standard error of the mean, $n=4$ per group. $\mathrm{P}>0.05$ vs. PT + vehicle. PT, photothrombosis
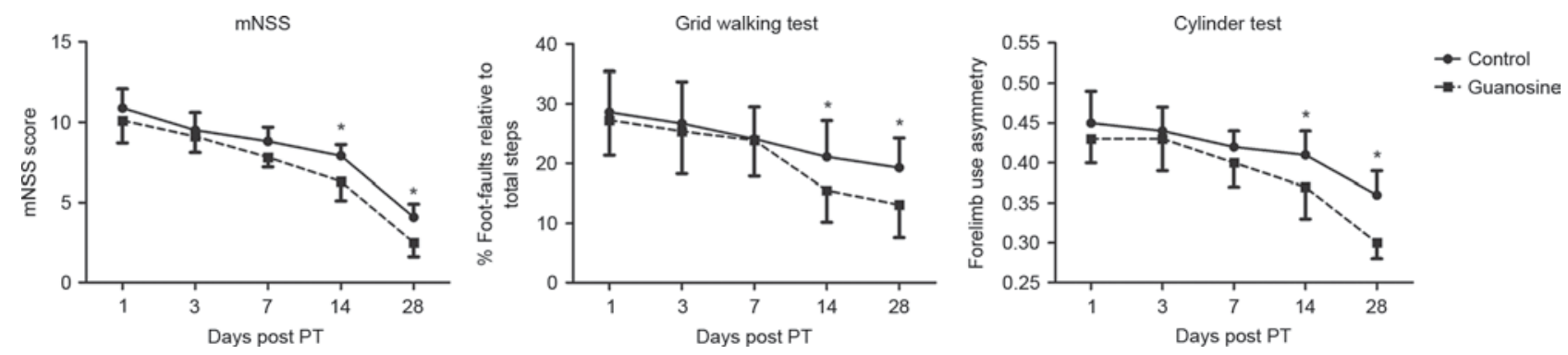

Figure 2. Delayed administration of guanosine improves long-term functional recovery following PT-induced stroke. No significant difference was detected on days 1 and 7 post-stroke in (A) mNSS score, (B) foot fault or (C) forelimb asymmetry between the vehicle group and guanosine group. However, a significant improvement in the mNSS score, foot fault and forelimb asymmetry was observed on days 14 and 28 post-stroke following guanosine treatment. Data are presented as the mean \pm standard error of the mean, $\mathrm{n}=8$ per group. ${ }^{*} \mathrm{P}<0.05$ vs. Control. PT, photothrombosis; mNSS, modified neurological severity scale.

Cruz Biotechnology, Inc.) and $\beta$-actin (catalogue no. 4970, dilution, 1:1,000; Cell Signalling Technology, Danvers, MA, USA). Subsequently, membranes were incubated with goat anti-rabbit horseradish peroxidase-conjugated IgG secondary antibody (catalogue no. AB501, dilution, 1:1,000; Novoprotein, Shanghai, China) for $2 \mathrm{~h}$ at room temperature. was used as a loading control for all experiments. Densitometry analysis was performed using the Image $\mathrm{J}$ software with normalization to $\beta$-actin.

Statistical analysis. All data are represented as mean \pm standard error of mean and were analysed using SPSS software (version 21.0; IBM SPSS, Armonk, NY, USA). The treatment effects on the behaviour score at different time points were analysed using repeated measures one-way analysis of variance, followed by Tukey-Kramer post hoc tests. When comparing two groups, statistical analysis of data was performed using Student's $t$-test. $\mathrm{P}<0.05$ was considered to indicate a statistically significant difference.

\section{Results}

Delayed administration of GUO does not reduce infarct volume but improved the post-stroke long-term functional outcome. There was no statistical difference in infarct volume on day 7 post-stroke between the two groups $(1.220 \pm 0.110 \mathrm{vs}$.
$1.125 \pm 0.120 \mathrm{~mm}^{3}, \mathrm{n}=8$ for each group, $\mathrm{P}>0.05$; Fig. 1 ). In addition, no difference was detected on days 1 and 7 post-stroke, in mNSS score, foot fault or forelimb asymmetry between the vehicle group and GUO group (Fig. 2A-C). However, a significant reduction in the mNSS score and foot fault was observed on days 14 and 28 post-stroke following GUO treatment $(\mathrm{P}<0.05$; Fig. 2A and B). Similarly, treatment with GUO significantly improved the function of the impaired forelimb on day 14 , and the effect was maintained up to 28 days post-stroke. The results indicated that delayed administration of GUO was able to promote long-term functional recovery following ischemic stroke (Fig. 2C).

Delayed administration of GUO enhances neurogenesis in the ischemic brain. GUO significantly increased the number of $\mathrm{BrdU}^{+}$cells in the ipsilateral SVZ and SGZ when compared with the vehicle group $(\mathrm{P}<0.05$; Fig. $3 \mathrm{~A}-\mathrm{C})$. These data indicated that administration of GUO enhances cell proliferation following stroke.

In addition, GUO significantly increased the number of $\mathrm{BrdU}^{+}$cells co-labelled with DCX, a marker of neural progenitor cells, in the ipsilateral SVZ and SGZ compared with the vehicle group $(\mathrm{P}<0.05$; Fig. $3 \mathrm{D}$ and $\mathrm{E})$ at all time points following stroke induction, suggesting that delayed administration of GUO promotes proliferation of neural progenitor cells in the SVZ and SGZ following stroke. 
A

Vehicle

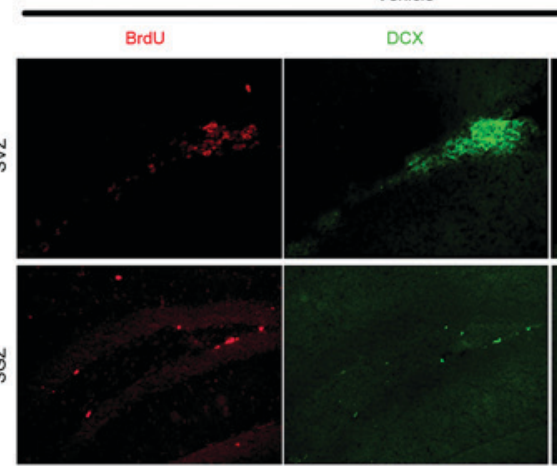

B

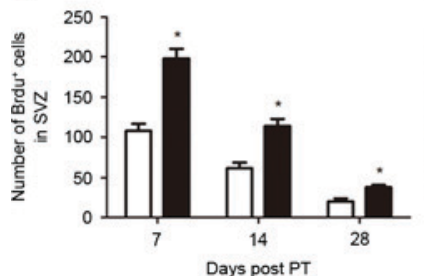

C

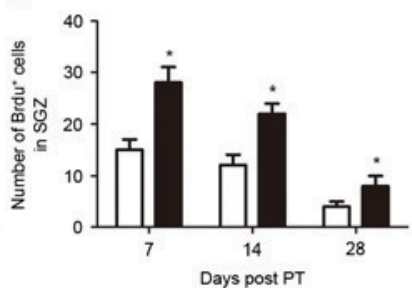

Merge
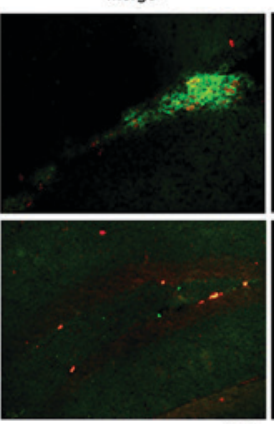

D

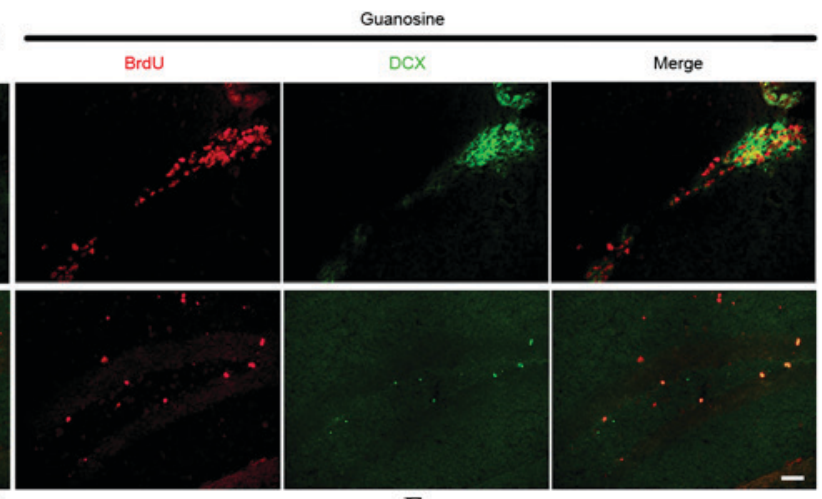

$\mathbf{E}$
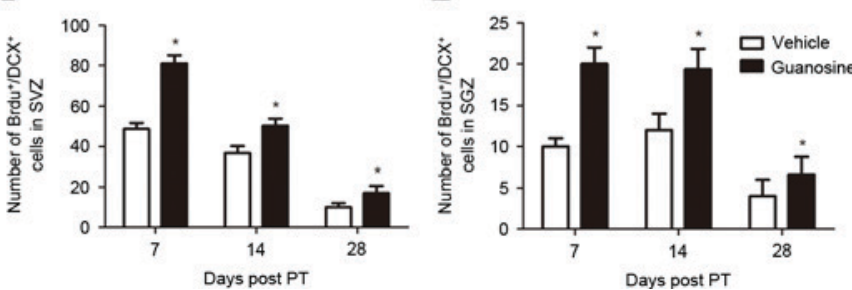

Figure 3. Delayed administration of guanosine promotes neural progenitor cells proliferation in the SVZ and SGZ. (A) Immunostaining of (red) BrdU and (green) DCX in the SVZ and SGZ of vehicle- and guanosine-treated mice at day 7 post-stroke. Quantification of BrdU ${ }^{+}$cells in the (B) SVZ and (C) SGZ, and $\mathrm{BrdU}^{+} / \mathrm{DCX}^{+}$cells in the (D) SVZ and (E) SGZ, at each time point for each group. Data are presented as the mean \pm standard error of the mean, $\mathrm{n}=6$ per group. ${ }^{*} \mathrm{P}<0.05$ vs. Vehicle. Scale, $100 \mu \mathrm{m}$. SVZ, subventricular zone; SGZ, subgranular zone; BrdU, bromodeoxyuridine; DCX, doublecortin.

To further investigate whether the proliferative neural progenitor cells is able to differentiate into functional neurons, double immunostaining was performed with NeuN (a marker of mature neurons) and BrdU. GUO significantly increased the number of $\mathrm{BrdU}^{+} / \mathrm{NeuN}^{+}$cells in the peri-infarction cortex following stroke, when compared with the vehicle group $(\mathrm{P}<0.05$; Fig. 4A and $\mathrm{B})$. These data indicated that GUO enhances the differentiation of new neural progenitor cells into mature neurons within the peri-infarction region following stroke.

Delayed administration of GUO enhances angiogenesis in the ischemic brain. To examine whether GUO influences the formation of new blood vessels in the ischemic brain, all blood vessels in the peri-infarction cortex were counted using vWF immunostaining. vWF is a vascular endothelial cell marker. GUO significantly increased vascular density in the peri-infarction cortex compared with the vehicle group $(\mathrm{P}<0.05$; Fig. 4C and D). Furthermore, these results presented a significant increase in the percentage of $\mathrm{BrdU}^{+} / \mathrm{vWF}^{+}$cells in mice treated with GUO, when compared with the vehicle group $(\mathrm{P}<0.05$; Fig. 4E). These data suggested that GUO enhances angiogenesis following stroke.

Delayed administration of GUO promotes the expression of neurotrophic factors. Western blot analysis demonstrated that GUO significantly increased the expression of VEGF and BDNF in the ischemic brain at day 14 post-stroke compared to the vehicle $(\mathrm{P}<0.05$; Fig. 5).

\section{Discussion}

The primary result of the present study was that delayed administration of GUO improved long-term functional outcome following a PT-induced stroke; however, did not reduce infarct volume. In addition, GUO enhanced post-ischemic neurogenesis and angiogenesis, which likely contributed to the restorative effects of GUO. Furthermore, GUO increased the expression of two key neurotrophins, BDNF and VEGF, suggesting that neurotrophic effects may contribute to the enhancing effects of GUO on post-ischemic neurogenesis and angiogenesis.

Treatment with GUO prior to or immediately following experimental cerebral ischemia confers acute neuroprotection in multiple in vitro and in vivo stroke models (10-14). The mechanisms responsible for the neuroprotective effects may be associated with the anti-oxidative stress, anti-excitatory toxicity and anti-apoptosis activities of GUO $(9,10,13,23)$. In the present study, delayed administration of GUO was investigated, to identify whether it improved long-term functional outcome following a stroke. The results indicated that GUO administered $24 \mathrm{~h}$ following PT accelerated long-term recovery. In particular, delayed GUO treatment only improved neurological functions from 14 days following the stroke and did not improve functions during the acute phase, which suggested that delayed GUO treatment may promote functional recovery through restorative rather than acute neuroprotective mechanisms.

In addition, the infarct volume at 7 days following stroke was not reduced. This result is consistent with previous studies in which infarct volume was only reduced by GUO when it was administered within a tight administration schedule $(11,12)$. These results suggest that delayed treatment with GUO did not exert an acute neuroprotective effect on cerebral ischemia, resulting in an unchanged infarct size.

GUO has been indicated to induce neurogenesis in SVZ in a mouse Parkinsonism model (24) and synaptogenesis in the healthy rat brain (18). However, whether GUO increases 
A
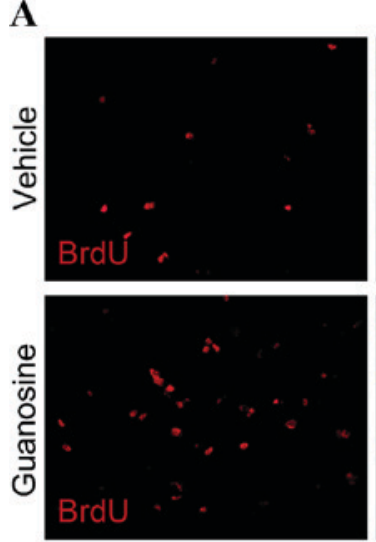

C
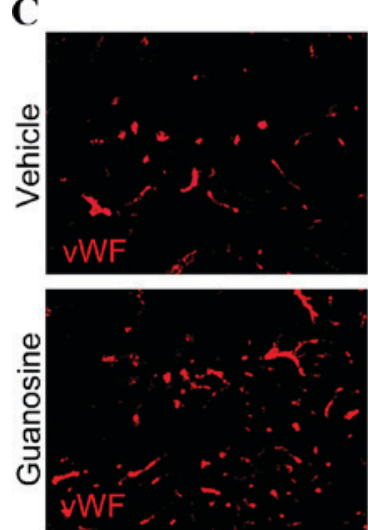
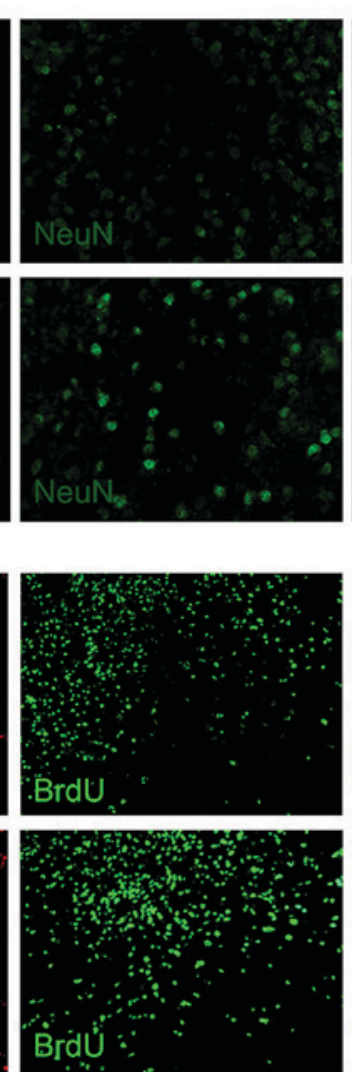
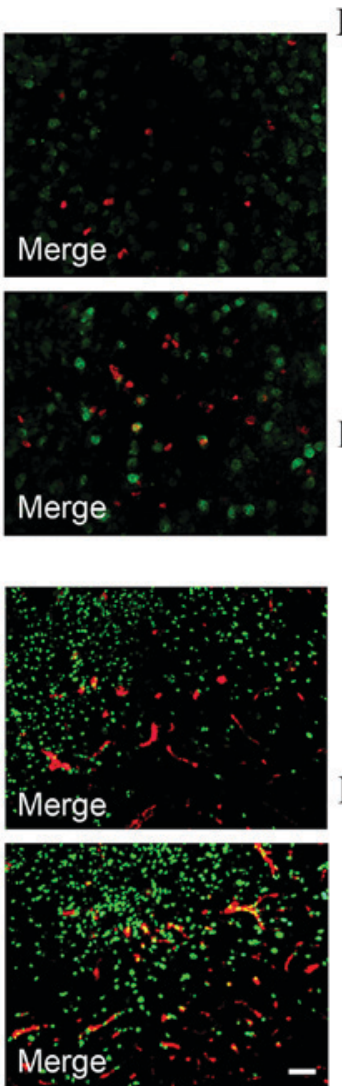
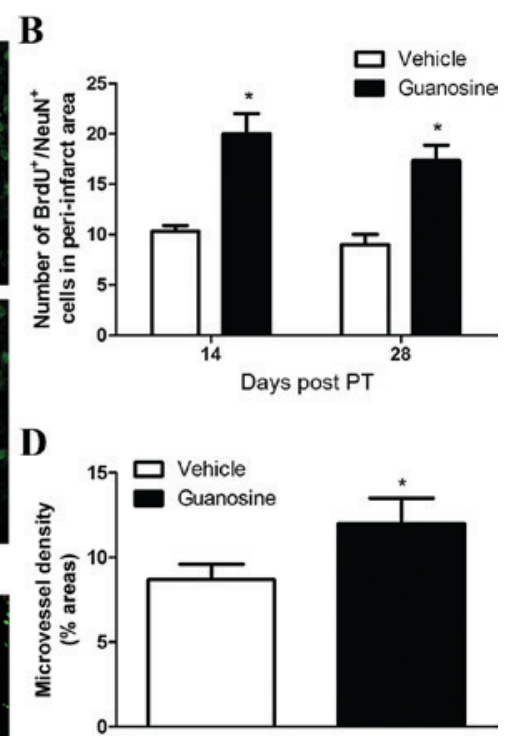

$\mathbf{E}$

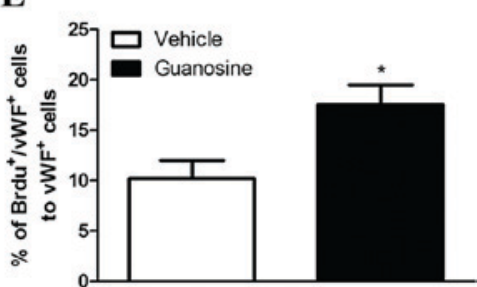

Figure 4. Delayed administration of guanosine increases the number of $\mathrm{BrdU}^{+} / \mathrm{NeuN}^{+}$cells and microvessel density within the peri-infarction region following stroke. (A) Immunostaining of (red) BrdU and (green) NeuN in the peri-infarct region of vehicle- and guanosine-treated mice at day 14 post-stroke. (B) Quantification of $\mathrm{BrdU}^{+} / \mathrm{NeuN}^{+}$cells in the peri-infarct region for each group. (C) Immunostaining of (red) vWF and (green) BrdU in the peri-infarct region of vehicle- and guanosine-treated mice at day 14 post-stroke. (D) microvessel density and (E) percentage of $\mathrm{BrdU}^{+} / \mathrm{vWF}^{+}$cells relative to total $\mathrm{vWF}^{+}$cells in the peri-infarct region for each group. Data are presented as the mean \pm standard error of the mean, $\mathrm{n}=6 \mathrm{per}$ group. * $\mathrm{P}<0.05 \mathrm{vs}$. Vehicle. Scale, $100 \mu \mathrm{m}$. BrdU, bromodeoxyuridine; NeuN, neuronal nuclei; vWF, von Willebrand factor.

A

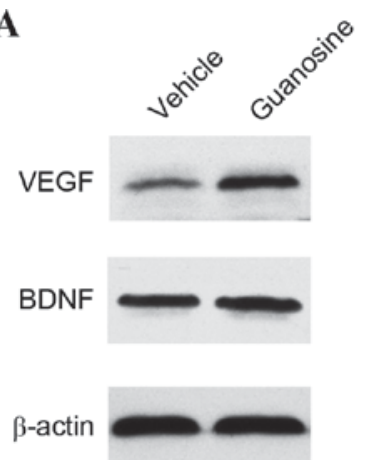

B

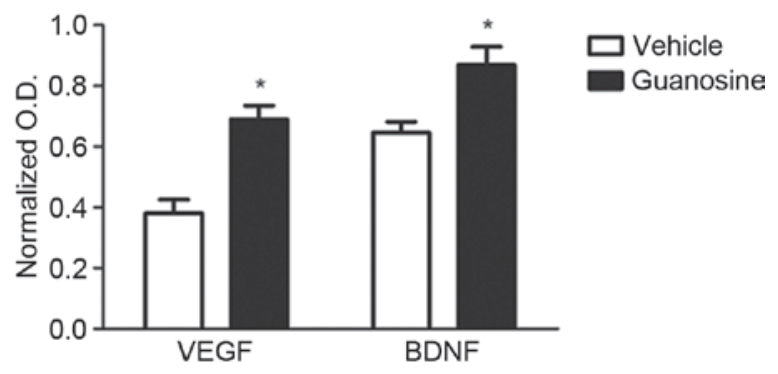

Figure 5. Delayed administration of guanosine increases the expression of VEGF and BDNF following stroke. (A) Representative western blots present levels of VEGF and BDNF in the ischemic brain 28 days following stroke. (B) Quantification (mean optical density, normalized to $\beta$-actin) of western blots. Data are presented as the mean \pm standard error of the mean, $n=6$ per group. ${ }^{*} \mathrm{P}<0.05$ vs. Control. VEGF, vascular endothelial growth factor; BDNF, brain-derived neurotrophic factor.

neurogenesis or angiogenesis post-stroke has never been studied, to the best of the authors' knowledge. GUO significantly increased the number of BrdU' cells in the SVZ and the SGZ, indicating that GUO promotes cell proliferation following stroke. As the number of $\mathrm{BrdU}^{+} / \mathrm{DCX}^{+}$cells increased in the $\mathrm{SVZ}$ in GUO-treated mice, GUO enhanced proliferation of endogenous neural progenitor cells. At 14 and 28 days post-stroke, treatment with GUO significantly increased the number of $\mathrm{BrdU}^{+}$ $\mathrm{NeuN}^{+}$cells in the peri-infarct region, when compared with the vehicle-treated group, suggesting that GUO promoted cell proliferation and the differentiation of new neural progenitor cells into mature neurons within the peri-infarction region. GUO was demonstrated to increase the microvessel density and $\mathrm{Brdu}^{+} / \mathrm{vwF}^{+}$cells in the peri-infarct region, when compared with the vehicle group, indicating angiogenesis post-stroke was enhanced and may contribute to neurological recovery.

Growth and neurotrophic factors have been demonstrated to promote neurogenesis and angiogenesis and improve 
neurological function following cerebral ischemia $(25,26)$. Previous in vitro studies have repeatedly demonstrated the neurotrophic effects of GUO (27). The present results further suggested that GUO significantly increased BDNF and VEGF levels in ipsilateral brain post-stroke. BDNF and VEGF are two important neurotrophic factors that have multiple effects on neurogenesis and angiogenesis, for example, they stimulate adult neurogenesis and promote migration of new neurons in the SVZ and dentate gyrus $(28,29)$. In addition, the expression of VEGF is associated with an increase in vascular density in the ischemic penumbra (30). Elevated BDNF and VEGF levels may contribute to the enhanced neurogenesis and angiogenesis by GUO. However, the causative link between them has not been investigated, therefore further studies are warranted.

In conclusion, delayed administration of GUO enhances neurogenesis and angiogenesis post-ischemic stroke and increases the expression of BDNF and VEGF. This contributes to improved long-term functional recovery.

\section{Acknowledgments}

The authors of the present study would like to thank Professor Di Song (Tongji Hospital, Tongji Medical College, Huazhong University of Science and Technology, Wuhan, China) for her comments on the manuscript.

\section{References}

1. Lozano R, Naghavi M, Foreman K, Lim S, Shibuya K, Aboyans V, Abraham J, Adair T, Aggarwal R, Ahn SY, et al: Global and regional mortality from 235 causes of death for 20 age groups in 1990 and 2010: A systematic analysis for the Global Burden of Disease Study 2010. Lancet 380: 2095-2128, 2012.

2. Lackland DT, Roccella EJ, Deutsch AF, Fornage M, George MG, Howard G, Kissela BM, Kittner SJ, Lichtman JH, Lisabeth LD, et al: Factors influencing the decline in stroke mortality: A statement from the American Heart Association/ American Stroke Association. Stroke 45: 315-353, 2014.

3. Adams HP Jr, Adams RJ, Brott T, del Zoppo GJ, Furlan A, Goldstein LB, Grubb RL, Higashida R, Kidwell C, Kwiatkowski TG, et al: Guidelines for the early management of patients with ischemic stroke: A scientific statement from the Stroke Council of the American Stroke Association. Stroke 34: 1056-1083, 2003.

4. Hermann DM and Chopp M: Promoting brain remodelling and plasticity for stroke recovery: Therapeutic promise and potential pitfalls of clinical translation. Lancet Neurol 11: 369-380, 2012.

5. Ming GL and Song H: Adult neurogenesis in the mammalian central nervous system. Annu Rev Neurosci 28: 223-250, 2005.

6. Liu J, Wang Y, Akamatsu Y, Lee CC, Stetler RA, Lawton MT and Yang GY: Vascular remodeling after ischemic stroke: Mechanisms and therapeutic potentials. Prog Neurobiol 115: 138-156, 2014.

7. Schmidt AP,Lara DR and Souza DO: Proposal of a guanine-based purinergic system in the mammalian central nervous system. Pharmacol Ther 116: 401-416, 2007.

8. Lanznaster D, Dal-Cim T, Piermartiri TC and Tasca CI: Guanosine: A neuromodulator with therapeutic potential in brain disorders. Aging Dis 7: 657-679, 2016.

9. Uemura Y, Miller JM, Matson WR and Beal MF: Neurochemical analysis of focal ischemia in rats. Stroke 22: 1548-1553, 1991.

10. Chang R, Algird A, Bau C, Rathbone MP and Jiang S: Neuroprotective effects of guanosine on stroke models in vitro and in vivo. Neurosci Lett 431: 101-105, 2008.

11. Rathbone MP, Saleh TM, Connell BJ, Chang R, Su C, Worley B, Kim M and Jiang S: Systemic administration of guanosine promotes functional and histological improvement following an ischemic stroke in rats. Brain Res 1407: 79-89, 2011.
12. Connell BJ, Di Iorio P, Sayeed I, Ballerini P, Saleh MC, Giuliani P, Saleh TM, Rathbone MP, Su C and Jiang S: Guanosine protects against reperfusion injury in rat brains after ischemic stroke. J Neurosci Res 91: 262-272, 2013.

13. Hansel G, Ramos DB, Delgado CA, Souza DG, Almeida RF, Portela LV, Quincozes-Santos A and Souza DO: The potential therapeutic effect of guanosine after cortical focal ischemia in rats. PLoS One 9: e90693, 2014.

14. Hansel G, Tonon AC, Guella FL, Pettenuzzo LF, Duarte T, Duarte MM, Oses JP, Achaval M and Souza DO: Guanosine protects against cortical focal ischemia. Involvement of inflammatory response. Mol Neurobiol 52: 1791-1803, 2015.

15. Gysbers JW and Rathbone MP: GTP and guanosine synergistically enhance NGF-induced neurite outgrowth from PC12 cells. Int J Dev Neurosci 14: 19-34, 1996.

16. Su C, Wang P, Jiang C, Ballerini P, Caciagli F, Rathbone MP and Jiang S: Guanosine promotes proliferation of neural stem cells through cAMP-CREB pathway. J Biol Regul Homeost Agents 27: 673-680, 2013

17. Jiang S, Khan MI, Lu Y, Wang J, Buttigieg J, Werstiuk ES, Ciccarelli R, Caciagli F and Rathbone MP: Guanosine promotes myelination and functional recovery in chronic spinal injury. Neuroreport 14: 2463-2467, 2003.

18. Gerrikagoitia I and Martínez-Millán L: Guanosine-induced synaptogenesis in the adult brain in vivo. Anat Rec (Hoboken) 292: 1968-1975, 2009.

19. Jang JY, Choi YW, Kim HN, Kim YR, Hong JW, Bae DW, Park SJ, Shin HK and Choi BT: Neuroprotective effects of a novel single compound 1-methoxyoctadecan-1-ol isolated from Uncaria sinensis in primary cortical neurons and a photothrombotic ischemia model. PLoS One 9: e85322, 2014.

20. Chen J, Sanberg PR, Li Y, Wang L, Lu M, Willing AE, Sanchez-Ramos J and Chopp M: Intravenous administration of human umbilical cord blood reduces behavioral deficits after stroke in rats. Stroke 32: 2682-2688, 2001.

21. Clarkson AN, Overman JJ, Zhong S, Mueller R, Lynch G and Carmichael ST: AMPA receptor-induced local brain-derived neurotrophic factor signaling mediates motor recovery after stroke. J Neurosci 31: 3766-3775, 2011.

22. de Vasconcelos Dos Santos A, da Costa Reis J, Diaz Paredes B Moraes L, Jasmin, Giraldi-Guimarães A and Mendez-Otero R: Therapeutic window for treatment of cortical ischemia with bone marrow-derived cells in rats. Brain Res 1306: 149-158, 2010.

23. Thomazi AP, Boff B, Pires TD, Godinho G, Battú CE, Gottfried C, Souza DO, Salbego C and Wofchuk ST: Profile of glutamate uptake and cellular viability in hippocampal slices exposed to oxygen and glucose deprivation: Developmental aspects and protection by guanosine. Brain Res 1188: 233-240, 2008.

24. Su C, Elfeki N, Ballerini P, D'Alimonte I, Bau C, Ciccarelli R, Caciagli F, Gabriele J and Jiang S: Guanosine improves motor behavior, reduces apoptosis, and stimulates neurogenesis in rats with parkinsonism. J Neurosci Res 87: 617-625, 2009.

25. Lichtenwalner RJ and Parent JM: Adult neurogenesis and the ischemic forebrain. J Cereb Blood Flow Metab 26: 1-20, 2006.

26. Zhao C, Deng W and Gage FH: Mechanisms and functional implications of adult neurogenesis. Cell 132: 645-660, 2008.

27. Rathbone MP, Middlemiss PJ, Gysbers JW, Andrew C, Herman MA, Reed JK, Ciccarelli R, Di Iorio P and Caciagli F: Trophic effects of purines in neurons and glial cells. Prog Neurobiol 59: 663-690, 1999.

28. Schabitz WR, Steigleder T, Cooper-Kuhn CM, Schwab S, Sommer C, Schneider A and Kuhn HG: Intravenous brain-derived neurotrophic factor enhances poststroke sensorimotor recovery and stimulates neurogenesis. Stroke 38: 2165-2172, 2007.

29. Jin K, Zhu Y, Sun Y, Mao XO, Xie L and Greenberg DA: Vascular endothelial growth factor (VEGF) stimulates neurogenesis in vitro and in vivo. Proc Natl Acad Sci USA 99: 11946-11950, 2002.

30. Hermann DM and Zechariah A: Implications of vascular endothelial growth factor for postischemic neurovascular remodeling. J Cereb Blood Flow Metab 29: 1620-1643, 2009. 